\title{
Effect of Different Environmental Stresses on the Antioxidant Capability of Air-Breathing Loach, Paramisgurnus dabryanus (Sauvage, 1878)
}

\author{
Yaqiu Liu ${ }^{1}$, Zhijian Wang ${ }^{2}$, Xinhui Li ${ }^{1 *}$, Jianrong $Z_{h a o^{2}}$ and Weitao Chen ${ }^{1}$ \\ ${ }^{1}$ Pearl River Fisheries Research Institute, Chinese Academy of Fishery Science, \\ Guangzhou, China \\ ${ }^{2}$ Key Laboratory of Freshwater Fish Reproduction and Development, Ministry of \\ Education, Southwest University, Chongqing 400715, China
}

\begin{abstract}
A B S T R A C T
The effect of oxidative stress on gill and gut enzyme activity in Paramisgurnus dabryanus was studied under different breathing restraint treatments. A total of 150 healthy mature individuals (mean initial body weight $=16.1 \pm 3.7 \mathrm{~g}$ and mean initial total fork length $=13.1 \pm 1.2 \mathrm{~cm}$ ) were selected for the experiment. The experimental specimens were separated into three modes: control group $(n=50)$ without any stress treatments; inhibited group $(n=50)$ with gut respiratory inhibition; and an air-exposed group $(\mathrm{n}=50)$ with gill respiratory inhibition. After a $7 \mathrm{~d}$ acclimation for the inhibited and air-exposed groups, individuals were returned to the same conditions as the control group for a further $3 \mathrm{~d}$ acclimation. The catalase (CAT) and superoxide dismutase (SOD) activity in the gill and gut in $P$. dabryanus were examined in all three groups after the $7 \mathrm{~d}$ stress acclimation, $1 \mathrm{~d}$ later, and after a $3 \mathrm{~d}$ recovery. It was found that CAT and SOD activity in the gill, foregut, and midgut in the air-exposed group were much lower than in the control group. After recovery for $1 \mathrm{~d}$, CAT activity in the gill and middle gut increased rapidly and was higher than in the inhibited and control groups. The SOD activity in the gill and gut also significantly increased, although there was no significant difference between the air-exposed and control groups. After a $3 \mathrm{~d}$ recovery, CAT and SOD activity in the gill and gut of $P$. dabryanus did not differ significantly between groups. There was a high viability of $P$. dabryanus under the air-exposed condition (in anaerobic conditions), while the level of oxidative stress in the gill, anterior, and middle gut clearly decreased. When recovering under normoxia conditions, the physical level of oxidative stress quickly returned to normal levels.
\end{abstract}

Article Information
Received 29 October 2019
Revised 02 December 2019
Accepted 27 December 2019
Available online 08 January 2021
Authors' Contribution
YL and ZW designed the study. YL
and JZ performed the experiments
and analyzed the data. ZW and WC
helped in experimental work and data
analysis. YL and XL wrote the article.
Key words
Air-exposed, Gut, Respiratory
inhibition, Oxidative stress,
Paramisgurnus dabryanus

\section{INTRODUCTION}

$\mathrm{T}$ he oxygen concentration in aquatic ecosystems has a direct impact on fish. It is often affected by diurnal changes in environmental factors, water temperature changes, and drought. Many fish are very sensitive to small changes in the oxygen concentration. To supplement oxygen consumption during aquatic hypoxia, some fish have evolved several adaptive strategies in terms of structure, physiology, and behavior (Catling et al., 2001; Gonzales et al., 2006; Huang et al., 2015). For example, members of the Loricariidae and the Callichthyidae families rely on specific tissues, such as vascularized stomach or gut tissue, for aerial gas exchange under hypoxic conditions (Bickler et al., 2007; Pelster et al., 2016).

It has been reported that there are more than 400 species of fish that have an accessory organ for air-breathing (Graham, 2011). Some accessory air-breathing organs are

\footnotetext{
* Corresponding author: Ixhui01@aliyun.com 0030-9923/2021/0002-0431 \$ 9.00/0

Copyright 2021 Zoological Society of Pakistan
}

located in the digestive tract, such as the esophagus, stomach and gut (Podkowa and Goniakowska-Witalinska, 2002). Gut air-breathing as an accessory respiratory approach are mainly subordinate to Cobitidae and Callichthyidae (Nelson, 2014). Studies of the gut airbreathing of fish such as Misgurnus anguillicaudatus (Goncalves et al., 2007), Lepidocephalichthys guntea (Moitra et al., 1989), Hoplosternum thoracatum (Huebner et al., 1978), Corydoras aeneus (Podkowa et al., 2002) have been reported. Paramisgurnus dabryanus relies on the gut for assisted respiration, and its accessory organs are located in the posterior part of the gut, which implies regional modifications of the gut for gas exchange. Like many other air-breathing fish, the respiratory structures of $P$. dabryanus are vascularized and have a thin diffusion distance between the external and internal mediums (Moitra et al., 1989; Goncalves et al., 2007).

Gut air-breathing, evolved as a physiological behavior and affects the fish's normal physiological and behavioral activities and its metabolic patterns and oxidative stress when repressed. The oxygen concentration required for aerobic metabolism may also cause the production 
of reactive oxygen species (ROS) (Pelster et al., 2016). The accumulation of ROS may result in oxidative stress, which is harmful for organisms (Lushchak, 2014). ROS concentrations vary with changes in oxygen availability, and organisms have developed sophisticated defense systems to eliminate reactive oxygen molecules when exposed to hypoxic and hyperoxic conditions (Welker et al., 2013). Hypoxia is one of the most common conditions that can cause oxidative stress (Welker et al., 2013). Catalase (CAT) and superoxide dismutase (SOD) play an important role in removing ROS. In addition, previous studies have clearly shown that the defense system against ROS is not static but is highly responsive to changing environmental conditions (Lushchak and Bagnyukova, 2006; Welker et al., 2013). For example, a significant increase in ROS degrading enzyme activities and in the concentration of antioxidants is frequently observed following periods of hypoxia.

However, the response of air-breathing fish to oxidative stress under different environmental stresses has not been reported in the literature. Therefore, we studied the changes of antioxidative capacity under water and respiratory gas respiration inhibition in separate treatments to determine the effect of environmental stress on oxidative stress in air-breathing fish. Basic adaptive evolutionary studies were consulted to provide basic information.

\section{MATERIALS AND METHODS}

\section{Experimental fish and holding conditions}

Loach (Paramisgurnus dabryanus) specimens used in the experiment were collected from a local market in Dianjian, Chongqing, China $\left(32^{\circ} 20^{\prime} \mathrm{N} ; 107^{\circ} 21^{\prime} \mathrm{E}\right)$. The body weight was $15.2 \pm 2.3 \mathrm{~g}$ and fork length was 13.3 $\pm 1.2 \mathrm{~cm}$. The experimental specimens were kept in a recirculating water tank $(180 \times 120 \times 80 \mathrm{~cm})$ in the laboratory for two weeks. In the tanks the fish had free access to air and could therefore breathe air voluntarily. During this period, the temperature of the de-chlorinated freshwater used was maintained at $21.3 \pm 1.7^{\circ} \mathrm{C}$ the oxygen level was kept above $7.0 \mathrm{mg} \mathrm{L}^{-1}$, the $\mathrm{pH}$ ranged from 6.7 to 7.5 . The photoperiod was maintained at $12 \mathrm{~h}$ light: $12 \mathrm{~h}$ dark and fish were fed with a commercial fish food (Xiwang Group, Chongqing, China).

\section{Experimental protocol}

At the end of domestication, 150 healthy fish of a similar size were selected and randomly divided into three groups (Fig. 1): (1) inhibited group $(\mathrm{n}=50)$, in which the $P$. dabryanus were kept active below the water surface for intestinal respiration; (2) air-exposed group $(\mathrm{n}=50)$, in which $P$. dabryanus were exposed to the air, although their bodies were kept; and (3) a control group, without any inhibition $(n=50)$. The details of the experimental protocol are shown in Figure 2.

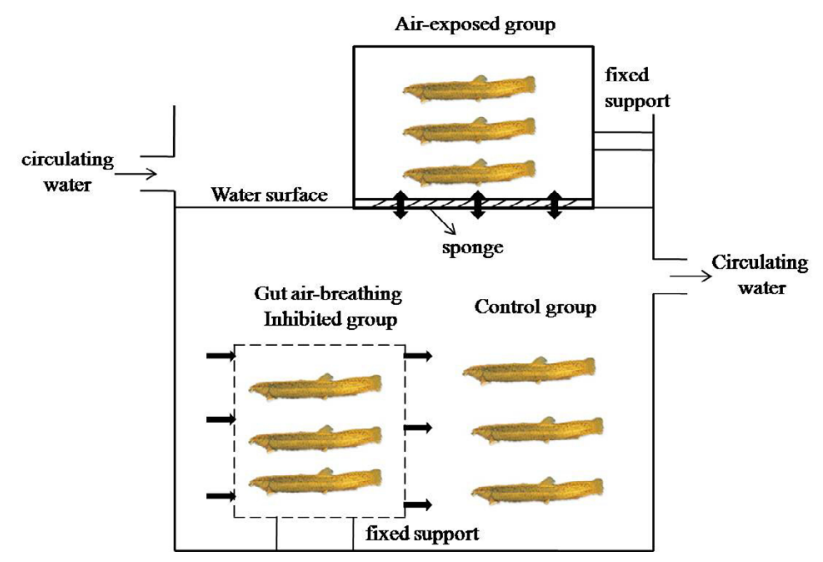

Fig. 1. Experimental device of P. dabryanus.

\begin{tabular}{|c|c|c|c|}
\hline Acclimation & & & \\
\hline 1st day & Normoxia & $\begin{array}{c}\text { Normoxia } \\
\text { (Gut air-breathing } \\
\text { restricted) }\end{array}$ & $\begin{array}{l}\text { Atmospheric } \\
\text { exposure } \\
\text { (Gill breathing } \\
\text { restricted) }\end{array}$ \\
\hline Recovery 1 day & Normoxia & Normoxia & Normoxia \\
\hline Recovery 3 days & Normoxia & Normoxia & Normoxia \\
\hline
\end{tabular}

Fig. 2. Illustration of the experimental protocol.

\section{Sample collection}

Six fish from each group were randomly selected for the determination of antioxidant enzyme activity. Body weight and fork length were measured after anesthesia with $0.1 \mathrm{~g} / \mathrm{L}$ ethyl m-aminobenzoate. The fish were placed on an ice tray and the gills, foregut, midgut, and hindgut were quickly removed into liquid nitrogen. The organs were then placed in a cryogenic refrigerator at $-80^{\circ} \mathrm{C}$ for the determination of further enzyme activity.

\section{Determination of enzyme activity}

The samples were removed from the refrigerator and placed on ice to thaw. After thawing was completed, each sample was weighed and homogenized. The homogenate was diluted with a F6 / 10 FLUKO hand-held 
Table I. Comparison of basic morphological parameters among different group (n=9).

\begin{tabular}{llll}
\hline & Control group & Inhibited group & Air-exposed group \\
\hline Weight $(\mathrm{g})$ & $15.90 \pm 4.29$ & $16.02 \pm 4.10$ & $16.19 \pm 3.24$ \\
Fork length $(\mathrm{cm})$ & $13.04 \pm 0.82$ & $12.35 \pm 1.20$ & $13.76 \pm 1.05$ \\
Gut mass $(\mathrm{g})$ & $0.27 \pm 0.08$ & $0.21 \pm 0.05$ & $0.25 \pm 0.06$ \\
Gut length $(\mathrm{cm})$ & $6.36 \pm 0.84$ & $5.47 \pm 0.62$ & $6.48 \pm 0.37$ \\
Gut length/fork length & $0.49 \pm 0.05$ & $0.45 \pm 0.06$ & $0.47 \pm 0.05$ \\
Gut mass/body mass & $0.017 \pm 0.01$ & $0.013 \pm 0.003$ & $0.016 \pm 0.004$ \\
Gill mass (g) & $0.25 \pm 0.02$ & $0.19 \pm 0.03$ & $0.20 \pm 0.02$ \\
Gill mass/body mass & $0.016 \pm 0.004$ & $0.014 \pm 0.005$ & $0.013 \pm 0.003$ \\
\hline
\end{tabular}

homogenizer for $5 \mathrm{~min}$ in an ice bath to ensure that the sample was fully ground. The volume ratio of the sample mass to the volume of the homogenate diluent was set to 1:9. The resulting homogenate was centrifuged using a cryogenic ultracentrifuge and the supernatant was used for the determination of antioxidant enzyme activity. CAT (EC1.11.1.6) and SOD (ECl.15.1.1) activity were measured in gill and gut tissue using a CAT assay kit (NO: A007, Nanjing Jiancheng Bioengineering Institute, PR China) and SOD assay kit (NO: A001, Nanjing Jiancheng Bioengineering Institute). All assays were performed on duplicate samples using a Thermo Multiskan spectrophotometer (Thermo Fisher Scientific, MA, USA). UV-permeable Corning 96-well microplates were used for all assays. All reactions were run at the saturating substrate concentrations determined for each enzyme ( $\mathrm{Li}$ et al., 2013).

\section{Statistical analysis}

IBM SPSS 19.0 was used for all statistical analysis of the recorded data. The effect of acclimation group (inhibited, air-exposed, control) and days on CAT and SOD activity were determined by a two-way analysis of variance (ANOVA). The effect of acclimation group (inhibited, air-exposed, control) on morphological parameters were determined by a one-way ANOVA followed by Dunnett's test. Any difference at $P<0.05$ was regarded as statistically significant. All values were expressed as mean $\pm \mathrm{SD}$.

\section{RESULTS}

\section{Morphological changes}

After domestication for $7 \mathrm{~d}$, there were no significant differences in the morphological parameters of $P$. dabryanus between groups (Table I).

\section{Gill antioxidant activity}

After domestication for $7 \mathrm{~d}$, the gill CAT activity in the air-exposed group was significantly lower than that in the control and inhibited group $(P<0.05)$, while there was no significant difference in CAT activity between the control and inhibited group (Fig. 3a). After recovery for $1 \mathrm{~d}$, the CAT activity in $P$. dabryanus was significantly increased in the air-exposed group $(P<0.05)$, which was higher than that in the control and inhibited groups $(P<$ 0.05 ) (Fig. 3a). After recovery for $3 \mathrm{~d}$, the CAT activity in $P$. dabryanus was significantly decreased $(P<0.05)$, and there was no significant difference in CAT activity between the two groups (Fig. 3a). The gill SOD activity in the airexposed group was much lower than that in the control and inhibited group $(P<0.05)$, while the SOD activity in $P$. dabryanus in the control and inhibited group were not significant different (Fig. 3b). After recovery for $1 \mathrm{~d}$, the SOD activity in $P$. dabryanus increased significantly in the air-exposed group $(P<0.05)$, and after recovery for $3 \mathrm{~d}$, there was no significant difference in SOD activity between the groups (Fig. 3b).

\section{Foregut antioxidant activity}

After domestication for $7 \mathrm{~d}$, the foregut CAT activity in $P$. dabryanus in the air- exposed group was much lower than that in the control and inhibited groups $(P<0.05)$ (Fig. 4a). The CAT activity in the foregut of $P$. dabryanus was significantly increased $(P<0.05)$ after recovery for 1 $\mathrm{d}$, and there was no significant difference in CAT activity between the groups (Fig. 4a). The SOD activity in $P$. dabryanus in the air-exposed group was lower than that in the control and inhibited groups $(P<0.05)$ (Fig. 4b). After a $1 \mathrm{~d}$ recuperation, the SOD activity in $P$. dabryanus was significantly increased $(P<0.05)$ in the air-exposed group and there was no significant difference in CAT activity between groups (Fig. 4b).

\section{Midgut antioxidant activity}

CAT activity in the midgut of $P$. dabryanus specimens in the air-exposed group was lower than that of the control group after domestication for $7 \mathrm{~d}(P<0.05)$ (Fig. 5a). After recovery for $1 \mathrm{~d}$, the CAT activity in the midgut of 
P. dabryanus sharply increased in the air-exposed group, and was much higher than that in the control and inhibited groups $(P<0.05)$ (Fig. 5a). The midgut SOD activity in $P$. dabryanus in the air-exposed group was significantly lower than that in the control and inhibited groups $(P<$ $0.05)$ (Fig. 5b). After a $1 \mathrm{~d}$ recovery, SOD activity was significantly increased $(P<0.05)$ in the midgut of $P$. dabryanus specimens that were exposed to air (Fig. 5b). After recovery for $3 \mathrm{~d}$, the SOD activity in $P$. dabryanus decreased significantly in the air-exposed group $(P<0.05)$. There was no significant difference in the CAT activity between groups (Fig. 5b).
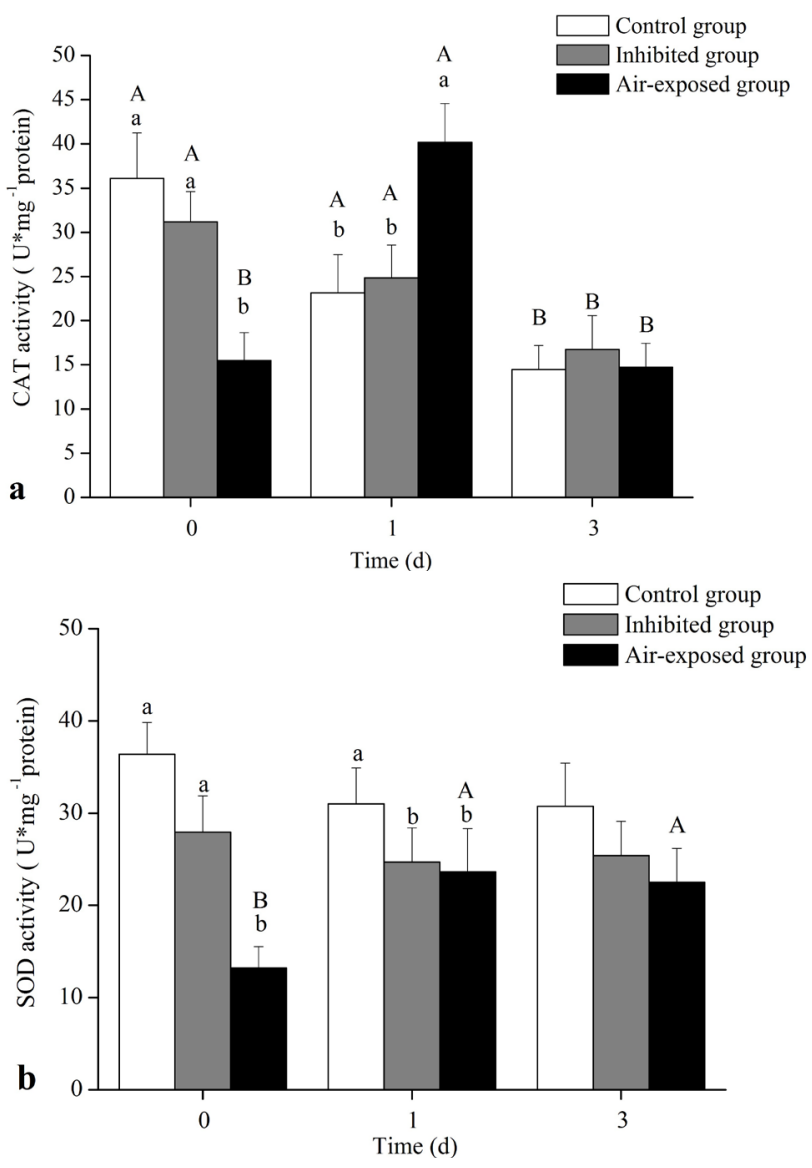

Fig. 3. Change of antioxidant capability in gill of loach. (a) catalase; (b) superoxide dismutase. Values in the table are represented as Mean $\pm \mathrm{SD} ; \mathrm{n}=6$; different superscripts (lowercase letters) indicate significant differences in different group; different superscripts (capital letters) indicate significant differences in different day; $P<0.05$

\section{Hindgut antioxidant activity}

After domestication for $7 \mathrm{~d}$, the CAT activity in the hindgut of $P$. dabryanus in the inhibited group was higher than that in the control and air-exposed groups $(P<0.05)$, and there was no significant difference in CAT activity between groups after recovery for $1 \mathrm{~d}$ (Fig. 6a). The SOD activity in $P$. dabryanus in the air-exposed group was lower than that in the control and inhibited groups $(P<$ 0.05 ) (Fig. 6b). SOD activity in $P$. dabryanus in the air exposed group increased rapidly after a $3 \mathrm{~d}$ recovery $(P<$ 0.05 ), and there was no significant difference in the SOD activity between groups (Fig. 6b).
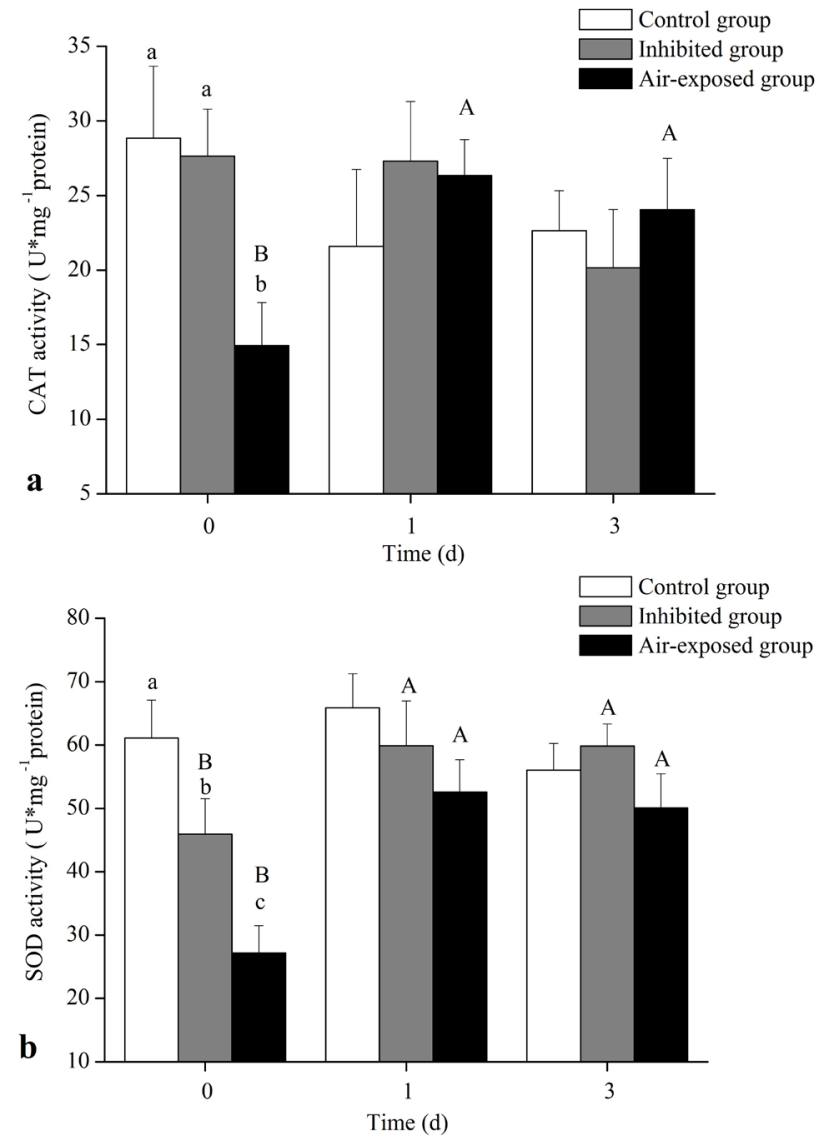

Fig. 4. Change of antioxidant capability in anterior intestine of loach. (a) catalase; (b) superoxide dismutase. Values in the table are represented as Mean $\pm \mathrm{SD} ; \mathrm{n}=6$; different superscripts (lowercase letters) indicate significant differences in different group; different superscripts (capital letters) indicate significant differences in different day, $P<0.05$

\section{DISCUSSION}

ROS production commonly accounts for 0.1$0.2 \%$ of the daily oxygen consumption in fish (Gorr et al., 2010). Large amounts of ROS can damage tissue structures (Costantini et al., 2010). Generally, the CAT and SOD systems are regarded as important cellular defense systems against oxidative stress. 

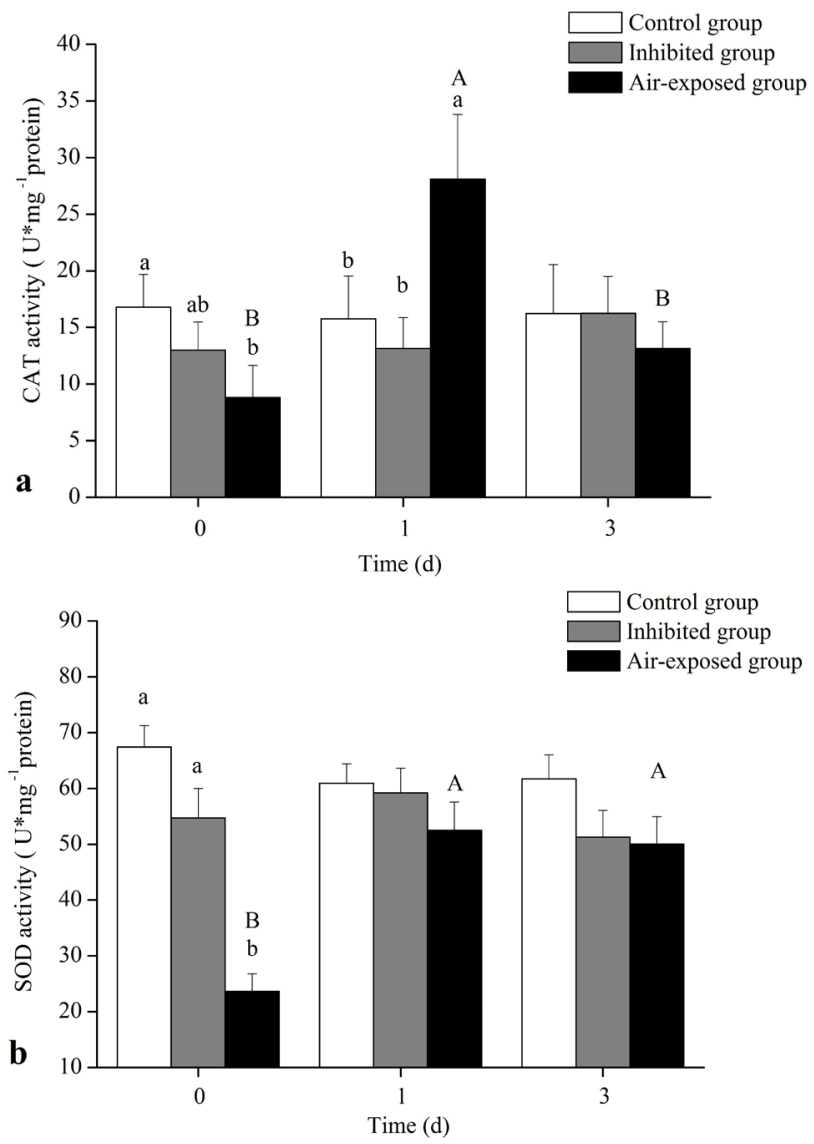

Fig. 5. Change of antioxidant capability in middle intestine of loach. (a) catalase; (b) superoxide dismutase. Values in the table are represented as Mean $\pm \mathrm{SD} ; \mathrm{n}=6$; different superscripts (lowercase letters) indicate significant differences in different group; different superscripts (capital letters) indicate significant differences in different day; $P<0.05$

We found that the air-breathing loach $P$. dabryanus can adjust the levels of oxidative stress it experiences when confronted with different environmental conditions. In recent studies, CAT and SOD activity in the gills, foregut, and midgut of $P$. dabryanus has been shown to decrease significantly when the fish are exposed to air. In contrast, in jeju (Hoplerythrinus unitaeniatus), the exposure of fish tissues to air may stimulate ROS production (Pelster et al., 2016). Under normal physiological conditions, organisms continually eliminate ROS and thus avoid damage to the body caused by oxidation. The mechanism of its elimination depends mainly on the body's antioxidant defense system, with CAT and SOD having important roles. We found that the CAT and SOD activity in the hindgut of $P$. dabryanus increased in the inhibited group. Studies have shown that when fish face stress conditions,
ROS are produced more than they are scavenged (Hur et al., 2001). The ROS content accumulates, which results in oxidative stress, leading to the body reaching a pathological state. Demple (1999) showed that in a body under chronic stress conditions the activity of antioxidant enzymes can increase to reduce oxidative damage.
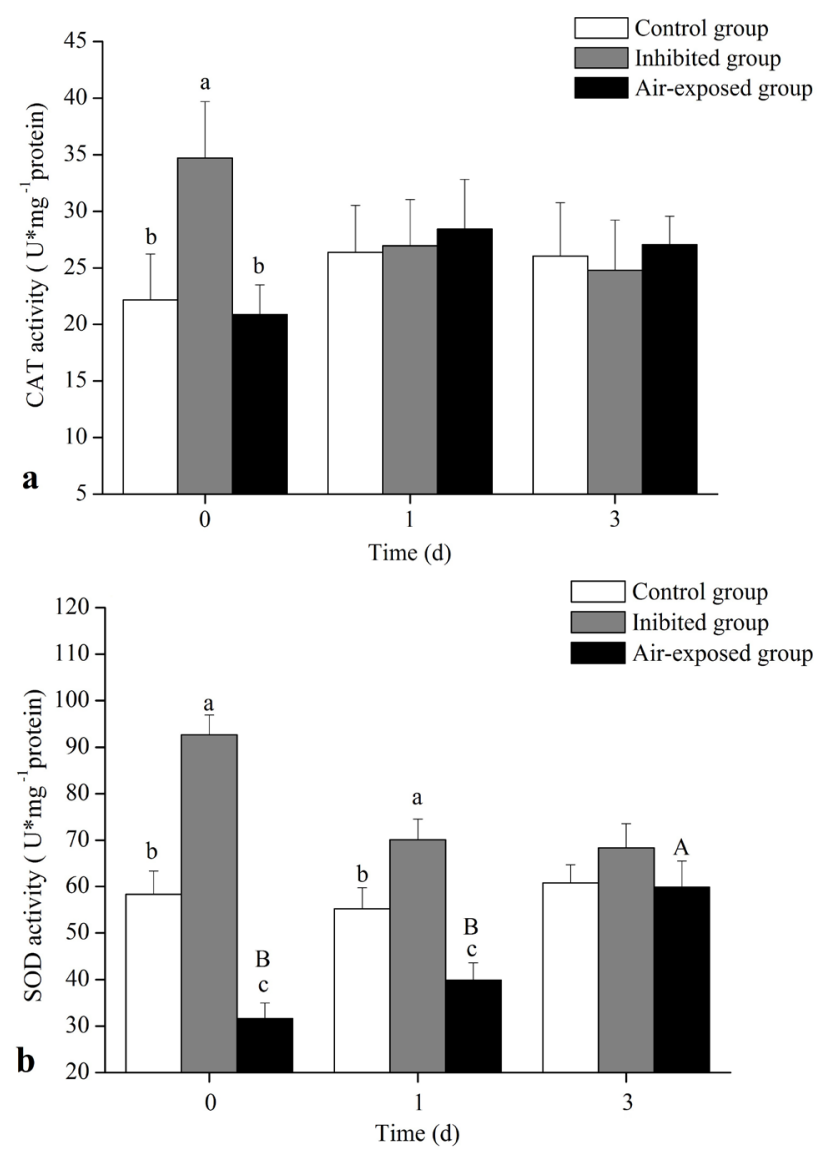

Fig. 6. Change of antioxidant capability in posterior intestine of loach. (a) catalase; (b) superoxide dismutase. Values in the table are represented as Mean $\pm \mathrm{SD}$; $\mathrm{n}=6$; different superscripts (lowercase letters) indicate significant differences in different group; different superscripts (capital letters) indicate significant differences in different day; $P<0.05$

When the fish were placed in normoxic conditions for $1 \mathrm{~d}$, the CAT and SOD activities were significantly increased, which may be due to the feedback regulation of dissolved oxygen in the environment. Some studies of the antioxidant system have observed an increased activity in antioxidant enzymes under hypoxic stress or following a return to normoxic levels (Costantini et al., 2010; Majmudar et al., 2010). Other studies have shown that fish are accompanied by significant oxidative stress when 
they recover from hypoxia, and some extremely hypoxiatolerant animals are more susceptible to oxidative stress during recovery than under hypoxia (Bickler and Buck, 2007).

With the re-introduction of oxygen, ROS may rapidly accumulate in tissues or cells. Excess ROS can cause oxidative stress and disorders of the antioxidant system (Lushchak et al., 2005). The results of this study are consistent with those of de Oliveira et al. (2005), who found that the antioxidant capacity of the body during reoxygenation may change significantly under hypoxia or stress, which may be an antioxidant strategy that organisms use to adapt to hypoxia and reoxygenation. After a $3 \mathrm{~d}$ resumption of normoxia, there was no significant difference in the antioxidant enzyme activity between groups. Under different environmental conditions, the fluctuation of the oxygen utilization rate in the matrix itself has an important effect on the ROS content (Welker et al., 2013). Pannunzio (1998) found that with the prolongation of hypoxia stress, the amount of oxygen in the body decreased and the SOD was reduced to $\mathrm{O}_{2}^{-}$reducing its activity in the body. When restored to normoxia for $1 \mathrm{~d}$, the CAT in the air exposed group was significantly increased and was higher than that in the control group. The aerobic capacity of the hindgut in the air exposure group and the inhibition group was similar to that of the control group. Different tissues have different adaptions to overcome environmental stress. These reactions are all distinctive anti-oxidant strategies that prevent the body's ROS from damaging itself.

\section{CONCLUSIONS}

Based on the above data, when $P$. dabryanus was exposed to the hypoxic and inhibited treatments, they had a high viability under the air-exposed condition (in anaerobic conditions), while the level of oxidative stress in the gill, foregut, and midgut clearly decreased. When exposed to normoxia conditions, $P$. dabryanus recovered quickly. The aquatic air-breathing fish $P$. dabryanus was able to regulate its physiological responses to the environmental disturbances provided in this study.

\section{ACKNOWLEDGEMENTS}

This work was financially supported by the Special Fund for Agro-scientific Research in the Public Interest under Grant number 201203086, Guangdong Province Natural Science Fund under Grant number 2016A030313147, Open Fund project of Key Laboratory of Freshwater Fish Reproduction and Development (Ministry of Education) under Grant number Klas-2018-01. National Natural Science Foundation of China under Grant number 31870527.
Statement of conflict of interest

Authors have declared no conflict of interest.

\section{REFERENCES}

Bickler, P.E. and Buck, L.T., 2007. Hypoxia tolerance in reptiles, amphibians, and fishes: life with variable oxygen availability. Annu. Rev. Physiol., 69: 145-170. https://doi.org/10.1146/annurev. physiol.69.031905.162529

Catling, D.C., Zahnle, K.J. and Mckay, C., 2001. Biogenic methane, hydrogen escape and the irreversible oxidation of early earth. Science, 293: 839-843. https://doi.org/10.1126/science.1061976

Costantini, D., Rowe, M., Butler, M.W. and McGraw, K.J., 2010. From molecules to living system: historical and contemporary issues in oxidative stress and antioxidant ecology. Funct. Ecol., 24: $950-959$. https://doi.org/10.1111/j.13652435.2010.01746.x

de Oliveira, U.O., da Rosa, Araújo, A.S., Belló-Klein, A., da Silva, R.S.M. and Kucharski, L.C., 2005. Effects of environmental anoxia and different periods of reoxygenation on oxidative balance in gills of the estuarine crab Chasmagnathus granulata. Comp. Biochem. Physiol., 140: 51-57. https://doi.org/10.1016/j.cbpc.2004.09.026

Demple B. Radical ideas: Genetic responses to oxidative stress. Clin. Exp. Pharmacol. P., 1999, 26: 64-68. https://doi.org/10.1046/j.1440-1681.1999.02993.x

Goncalves, A., Castro, L., Pereira-Wilson, C., Coimbra, J. and Wilson, J., 2007. Is there a compromise between nutrient uptake and gas exchange in the gut of Misgurnus anguillicaudatus, an intestinal airbreathing fish? Comp. Biochem. Physiol., 2: 345355. https://doi.org/10.1016/j.cbd.2007.08.002

Gonzales, T.T., Katoh, M. and Ishimatsu, A., 2006. Air breathing of aquatic burrow-dwelling eel goby, Odontamblyopus lacepedii (Gobiidae: Amblyopinae). J. exp. Biol., 209: 1085-1092. https://doi.org/10.1242/jeb.02092

Gorr, T.A., Wichmann, D., Hu, J., Hermes-Lima, M., Welker, A.F., Terwilliger, N., Wren, J.F., Viney, M., Morri, S., Nilsson, G.E., Deten, A., Soliz, J. and Assmann, M., 2010. Hypoxia tolerance in animals: biology and application. Physiol. Biochem. Zool., 83: 733-752. https://doi.org/10.1086/648581

Graham, J.B., 2011. The biology, diversity, and natural history of air-breathing fishes: an introduction. In: Encyclopedia of fish physiology (ed. A.P. Farrell), Academic Press, New York. pp. 1850-1860. https:// doi.org/10.1016/B978-0-12-374553-8.00044-7 
Huang, C.Y., Lin, H.H., Lin, C.H. and Lin, C.H. 2015. The absence of ion-regulatory suppression in the gills of the aquatic air-breathing fish Trichogaster lalius during oxygen stress. Comp. Biochem. Physiol., 179: 7-16. https://doi.org/10.1016/j. cbpa.2014.08.017

Huebner, E. and Chee, G., 1978. Histological and ultrastructural specialization of the digestive tract of the intestinal air breather Hoplosternum thoracatum (Teleost). J. Morphol., 157: 301-327. https://doi.org/10.1002/jmor.1051570305

Hur, E., Chang, K. Y., Lee. E., Lee., S. K. and Park, H. 2001. Mitogen-activated protein kinase kinase inhibitor PD98059 blocks the trans-activation but not the stabilization or DNA binding ability of hypoxia-inducible factor-1 alpha. Mol. Pharmacol., 59: 1216-1224. https://doi.org/10.1124/ mol.59.5.1216

Li, X.M., Yu, L.J., Wang, C., Zeng, L.Q., Cao, Z.D., Fu, S.J. and Zhang, Y.G. 2013. The effect of aerobic exercise training on growth performance, digestive enzyme activities and postprandial metabolic response in juvenile qingbo (Spinibarbus sinensis). Comp. Biochem. Physiol., 166: 8-16. https://doi. org/10.1016/j.cbpa.2013.04.021

Lushchak, V.I., 2014. Free radicals, reactive oxygen species, oxidative stress and its classification. Chem. Biol. Interact., 224:164-175. https://doi. org/10.1016/j.cbi.2014.10.016

Lushchak, V.I. and Bagnyukova, T.V., 2006. Effects of different environmental oxygen levels on free radical processes in fish. Comp. Biochem. Physiol., 144: 283-289. https://doi.org/10.1016/j. cbpb.2006.02.014

Lushchak, V.I., Bagnyukova, T.V., Lushchak, O.V., Storey, J.M. and Storey, K.B. 2005. Hypoxia and recovery perturb free radical processes and antioxidant potential in common carp (Cyprinus carpio) tissues. Int. J. Biochem. Cell, 37: 13191330. https://doi.org/10.1016/j.biocel.2005.01.006

Majmudar, A.J., Wong, W.J. and Simon, M.C., 2010. Hypoxia-inducible factors and the response to hypoxic stress. Mol. Cell, 40: 294-309. https://doi. org/10.1016/j.molcel.2010.09.022

Moitra, A., Singh, O.N. and Munshi, J.S.D., 1989. Microanatomy and cytochemistry of the gastrorespiratory tract of an air-breathing cobitidid fish, Lepidocephalichthys guntea. Ichthyol. Res., 36: 227-231. https://doi.org/10.1007/BF02914326

Nelson, J.A., 2014. Breaking wind to survive: fishes that breathe air with their gut. J. Fish Biol., 84: 554-576. https://doi.org/10.1111/jfb.12323

Pannunzio, T.M. and Storey, K.B., 1998. Antioxidant defenses and lipid peroxidation during anoxia stress and aerobic recovery in the marine gastropod Littorina littorea. J. exp. Mar. Biol. Ecol., 221: 277-292. https://doi.org/10.1016/S00220981(97)00132-9

Pelster, B., Giacomin, M., Wood, C.M. and Val, A.L. 2016. Improved ROS defense in the swimbladder of a facultative air-breathing erythrinid fish, jeju, compared to a non-air-breathing close relative, traira. J. comp. Physiol., 186: 615-624. https://doi. org/10.1007/s00360-016-0981-5

Podkowa, D. and Goniakowskawitalińska, L., 2002. Adaptations to the air breathing in the posterior intestine of the catfish (Corydoras aeneus, Callichthyidae). A histological and ultrastructural study. Folia Biol., 50: 69-82.

Sun, H.J., Li, J.J., Tang, L.S. and Zhou, Y., 2012. Responses of crucian carp Carassius auratus to long-term exposure to nitrite and low dissolved oxygen levels. Biochem. Syst. Ecol., 44: 224-232. https://doi.org/10.1016/j.bse.2012.06.011

Welker, A.F., Moreira, D.C., Campos, É.G. and Hermes-Lima, M. 2013. Role of redox metabolism for adaptation of aquatic animals to drastic changes in oxygen availability. Comp. Biochem. Physiol., 165: 384-404. https://doi.org/10.1016/j. cbpa.2013.04.003

Willmore, W.G. and Storey, K.B., 1997. Antioxidant systems and anoxia tolerance in a freshwater turtle Trachemys scripta elegans. Mol. Cell. Biochem., 170: $177-185$. 